\title{
Interacting Fock Spaces and Orthogonal Polynomials in several variables
}

\author{
Luigi Accardi, Marcolino Nahni
}

October 1, 2006

\author{
Centro Vito Volterra, Università di Roma "Tor Vergata" \\ e-mail: accardi@volterra.mat.uniroma2.it, WEB page: http://volterra.mat.uniroma2.it
}

\begin{abstract}
We extend to polynomials in several variables the Accardi-Bozeiko canonical isomorphism between 1-mode interacting Fock spaces and orthogonal polynomials in one variable. This gives a constructive rule to write down easily the quantum decomposition, as a sum of creation, annihilation and number operators, of an arbitrary vector valued random variable with moments of any order. In the multi-mode case not all interacting Fock spaces are canonically isomorphic to spaces of orthogonal polynomials. We characterize those which enjoy this property in terms of a sequence of quadratic commutation relations among finite dimensional matrices.
\end{abstract}




\section{Introduction}

In the past years the theory of interacting Fock spaces has been used in a multiplicity of different contexts (cf. [Hash97], [HaHoOb01],[HaObTa01], [Asai01], [Das01a], [Das01b]). In many of these papers the natural correspondence between interacting Fock spaces and orthogonal polynomials played a relevant role.

This correspondence was proved, for one-mode interacting Fock spaces by Accardi and Bozeiko who shewed that the theory of 1-mode interacting Fock spaces is canonically isomorphic to the theory of orthogonal polynomials in one variable, i.e. with respect to a probability measure on the real line with finite moments of any order. The canonical feature of this isomorphism is exhibited by the fact that it maps the multiplication operator by the independent variable into a linear combination of the creation, annihilation and number operators of the corresponding interacting Fock space.

The problem to extend this isomorphism to polynomials in several variables has been open for a few years. In the present paper we discuss a solution of this problem. The main new feature with respect to the one--mode case is that, in the multi-mode case, case not all interacting Fock spaces are canonically isomorphic to spaces of orthogonal polynomials. We characterize those which enjoy this property in terms of a sequence of quadratic commutation relations among finite dimensional matrices. This gives in particular a constructive rule to write down easily the quantum decomposition, as a sum of creation, annihilation and number operators, of an arbitrary vector valued random variable with moments of any order.

Let $d \in \mathbf{N}$. We denote $\mu$ a probability measure on $\mathbf{R}^{d}$ with finite moments of any order; $x=\left(x_{1}, \ldots, x_{d}\right)$ any element of $\mathbf{R}^{d} ; x_{j}$ denote the coordinates in the canonical basis; $X^{0}=\left(X_{1}^{0}, \ldots, X_{d}^{0}\right):\left(\mathbf{R}^{d}, \mu\right) \rightarrow \mathbf{R}^{d}$ the $\mathbf{R}^{d}$-valued coordinate random variable, characterized by:

$$
X_{j}^{0}(x)=x_{j} \quad ; \quad j=1, \ldots, d
$$

Definition 1 The complex $*$-algebra $\mathcal{P}=\mathcal{P}^{d}$, with identity, generated (algebraically) by the $X_{j}^{0}(j=1, \ldots, d)$ and the constant functions with pointwise addition and multiplication and $f^{*}(x)=\bar{f}(x)$-complex conjugate) is called the "polynomial algebra in d indeterminates".

In the following, the dimension $d$ will be fixed, so we will frequently omit the super-script $d$ and write, for example, $\mathcal{P}$ instead of $\mathcal{P}^{d}$. 
The $\mu$-integral defines a state on $\mathcal{P}$ and the $G N S$ construction, applied to the pair $\{\mathcal{P}, \mu\}\left[\right.$ Sak71] gives a Hilbert space $\mathcal{H}_{\mu}$, a representation

$$
\pi_{\mu}: \mathcal{P} \rightarrow \mathcal{B}\left(\mathcal{H}_{\mu}\right)
$$

(the bounded linear operators on $\mathcal{H}_{\mu}$ ) and a unit vector $1_{\mu}$, cyclic for $\pi(\mathcal{P})$. $\mathcal{H}_{\mu}$ is a closed sub-space of $L^{2}\left(\mathbf{R}^{d}, \mu\right)$ and coincides with it if and only if the measure $\mu$ is uniquely determined by its moments. Thus the space $\mathcal{H}_{\mu}$ gives in some sense a measure of the non uniqueness of the moment problem for $\mu$.

Definition 2 We will say that $\mu$ is generic if

(i) the union of the coordinate hyperplanes $x_{j}=0(j=1, \ldots, d)$ has $\mu$-measure zero

(ii) $\pi_{\mu}$ is injective (notice that this implies that also the map $p \in \mathcal{P} \rightarrow$ $\pi_{\mu}(p) \cdot 1_{\mu}$ is injective).

This is surely the case if $\pi_{\mu}$ has a density with respect to the Lebesgue measure. In the generic case the $*$-algebra

$$
\mathcal{P}_{\mu}:=\pi(\mathcal{P})
$$

is isomorphic to $\mathcal{P}$ and also the elements of $\mathcal{P}_{\mu}$ will be called polynomials. They are bounded operators on $\mathcal{H}_{\mu}$ if and only if $\mu$ has compact support.

Define the coordinate multiplication operators

$$
X_{j}^{0} f(x):=x_{j} f(x) ; \quad j=1, \ldots, d
$$

and their action on $\mathcal{H}_{\mu}$ :

$$
\pi\left(X_{j}^{0}\right) \mid \mathcal{H}_{\mu}=: X_{j} \quad ; \quad j=1, \ldots, d
$$

Condition (i) above implies that the operators $X_{j}(j=1, \ldots, d)$ are invertible in $\mathcal{H}_{\mu}$. In the following we will identify the elements of $\mathcal{H}_{\mu}$ to elements of $L^{2}\left(\mathbf{R}^{d}, \mu\right)$ so that, for each $j=1, \ldots, d, X_{j}$ acts by multiplication on $\mathcal{H}$ :

$$
X_{j} f(x)=x_{j} f(x) ; f \in \mathcal{H}
$$

and defines a symmetric pre-closed operator on $\mathcal{H}$ on the dense invariant domain $\mathcal{P}_{\mu} \cdot 1_{\mu}$. The algebra $\mathcal{P}_{\mu}$ is generated by the monomials

$$
X_{1}^{n_{1}} \ldots X_{d}^{n_{d}} ; \quad \forall n_{1}, \ldots, n_{d} \in \mathbf{N}
$$


and the vectors

$$
X_{1}^{n_{1}} \ldots X_{d}^{n_{d}} \cdot 1_{\mu}
$$

are total in $\mathcal{H}_{\mu}$ by construction.

In the following, when no confusion can arise, we shall denote with the same symbol both the vectors (5) and the corresponding multiplication operators (4) in $\mathcal{P}_{\mu}$.

Definition 3 For each $n \in \mathbf{N}$ define

$$
\mathcal{P}_{n}^{d}:=\text { linear span of }\left\{X_{1}^{n_{1}} \ldots X_{d}^{n_{d}} ; n_{j} \in \mathbf{N}, \quad \sum_{j=1}^{d} n_{j} \leq n\right\}
$$

It is clear that $\mathcal{P}_{n}^{d}$ is the vector space of all polynomials in the variables $X_{1}, \ldots, X_{d}$ of degree at most $n$, where the degree of the monomial $X_{1}^{n_{1}} \ldots X_{d}^{n_{d}}$ is $\sum_{j=1}^{d} n_{j}$ and the degree of a polynomial $P$ is defined to be the highest, among the degrees of the monomials which appear in $P$ with a non zero coefficient. Notice that, for each $j=1, \ldots, d$,

$$
X_{j} \mathcal{P}_{n}^{d} \subseteq \mathcal{P}_{n+1}^{d}
$$

Define inductively

$$
\begin{gathered}
V_{0}=\mathbf{C} \cdot 1_{\mu}=\mathcal{P}_{0}^{d} \cdot 1_{\mu} \\
V_{n+1}^{d}:=\left(\bigoplus_{k=0}^{n} V_{k}^{d}\right)^{\perp} \cap \mathcal{P}_{n+1}^{d} \cdot 1_{\mu}
\end{gathered}
$$

Lemma 1 . For each $n \in \mathbf{N}$ :

$$
\mathcal{P}_{n}^{d} \cdot 1_{\mu}=\bigoplus_{k=0}^{n} V_{k}^{d}
$$

In particular:

$$
V_{n+1}^{d}=\left(\bigoplus_{k=0}^{n} V_{k}^{d}\right)^{\perp} \cap\left\{\text { linear span of } X_{1}^{n_{1}} \ldots X_{d}^{n_{d}} \text { with }: n_{j} \in \mathbf{N},(j=1, \ldots, d), \sum_{j=1}^{d} n_{j}=n+1\right\}
$$




\section{Proof. Clear.}

For $n_{1}+\ldots+n_{d}=n$, denote $: X_{1}^{n_{1}} \ldots X_{d}^{n_{d}}$ : the orthogonal projection of $X_{1}^{n_{1}} \ldots X_{d}^{n_{d}} \cdot 1_{\mu}$ in $\bigoplus_{k<n} V_{k}=\mathcal{P}_{n-1} \cdot 1_{\mu}$. By linearity the symbol $: p_{n}(X):$ is defined for any polynomial $p_{n}$ of degree $n$.

The vectors : $X_{1}^{n_{1}} \ldots X_{d}^{n_{d}}$ : span $V_{n}$ but in general, they are not orthogonal.

Lemma 2 . (Linear independence) For any fixed $n$, the vectors : $X_{1}^{n_{1}} \ldots X_{d}^{n_{d}}$ :, with $\sum_{j}^{d} n_{j}=n$, are linearly independent. In particular $V_{n}^{d} \equiv C^{r_{n}^{d}}$ with

$$
r_{n}^{d}:=\left(\begin{array}{c}
n+d-1 \\
n
\end{array}\right)
$$

and the vectors : $X_{1}^{n_{1}} \ldots X_{d}^{n_{d}}$ : are a basis of $V_{n}^{d}$.

Proof. Fix $n \in \mathbf{N}$. The genericity of $\mu$ implies that the map $p_{n} \mapsto: p_{n}$ : is injective $\left(p_{n}\right.$ polynomial of degree $\left.n\right)$. Therefore, $\forall n_{j} \in \mathbf{N}$ with $\sum_{j}^{d} n_{j}=$ $n$, the : $X_{1}^{n_{1}} \ldots X_{d}^{n_{d}}$ : are linearly independent because, by the genericity condition, such are the $X_{1}^{n_{1}} \ldots X_{d}^{n_{d}} \cdot 1_{\mu}$. Therefore the cardinality of the set

$$
\left\{: X_{1}^{n_{1}} \ldots X_{d}^{n_{d}}:, n_{j} \in \mathbf{N}, \sum_{j}^{d} n_{j}=n\right\}
$$

is $\left(\begin{array}{c}n+d-1 \\ n\end{array}\right)$. Since $\operatorname{dim} \mathcal{P}_{n}^{d}=\left(\begin{array}{c}n+d \\ d\end{array}\right)$ [KrSh67], using the identity

$$
\left(\begin{array}{c}
n+d \\
d
\end{array}\right)-\left(\begin{array}{c}
n+d-1 \\
d
\end{array}\right)=\left(\begin{array}{c}
n+d-1 \\
n
\end{array}\right)
$$

we can conclude that

$$
\operatorname{dim} V_{n}^{d}=\operatorname{dim} \mathcal{P}_{n}^{d}-\operatorname{dim} \mathcal{P}_{n-1}^{d}=\left(\begin{array}{c}
n+d-1 \\
n
\end{array}\right)
$$

This proves the lemma, since the set $\left\{: X_{1}^{n_{1}} \ldots X_{d}^{n_{d}}:, n_{j} \in \mathbf{N}, \sum_{j}^{d} n_{j}=n\right\}$ generates $V_{n}^{d}$.

Remark. Since the elements of any orthonormal basis of $V_{n}$ are in one-to-one correspondence with the solutions (in $\mathbf{N}$ ) of the equation

$$
n_{1}+\ldots+n_{d}=n
$$


we will often use the notation

$$
|\bar{n}>=| n_{1}, \ldots, n_{d}>
$$

to denote an arbitrary orthonormal basis of $V_{n}$. When confusion can arise we will use the more explicit notation

$$
\phi_{\bar{n}}=\phi_{n_{1}, \ldots, n_{d}}
$$

The recurrence relations among orthogonal polynomials are consequences of the following result:

Theorem 1 . For any $n \in \mathbf{N}$, if $k \notin\{n-1, n, n+1\}$ then for each $j=1, \ldots, d$,

$$
X_{j} V_{n} \perp V_{k}
$$

Proof. Let $\mid \bar{n}>\in V_{n}$ and $f \in \mathcal{P}_{n-2}$, then for each $j=1, \ldots, d, \quad X_{j} f \in$ $P_{n-1}^{d}$ hence, by the symmetry of $X_{j}$ and (6):

$$
<X_{j} \bar{n}, f>=<\bar{n}, X_{j} f>=0
$$

because by assumption $\mid \bar{n}>\in V_{n} \perp \mathcal{P}_{n-1}$.

If $f \in V_{k}$ with $k>n+1$, then $f \perp X_{j} \mid \bar{n}>$ because $X_{j} \mid \bar{n}>$ is a polynomial of degree $n+1$. This proves the statement.

Denote $P_{m}$ the orthogonal projection on $V_{m}$. The following is a multidimensional generalization of the Jacobi relations for 1-dimensional orthogonal polynomials.

Corollary 1 (Recurrence relations) Let us fix, for any $n \in \mathbf{N}$, an orthonormal basis $\left\{|\bar{n}\rangle=\mid n_{1}, \ldots, n_{d}>; \sum_{j}^{d} n_{j}=n\right\}$ of $V_{n}$. For each $\bar{n}=$ $\left(n_{1}, \ldots, n_{d}\right) \in \mathbf{N}^{d}$ with $\sum_{j}^{d} n_{j}=n$, and for each $j=1, \ldots, d$ we have

$$
X_{j} P_{n}=P_{n+1} X_{j} P_{n}+P_{n} X_{j} P_{n}+P_{n-1} X_{j} P_{n}
$$

Proof. We know from Theorem 1 that for each $j=1, \ldots, d, n \in \mathbf{N}$, $\mid \bar{n}>\in V_{n}$,

$$
X_{j} \mid \bar{n}>\in V_{n+1} \oplus V_{n} \oplus V_{n-1}
$$

Since $P_{n+1}+P_{n}+P_{n-1}$ is the orthogonal projection on $V_{n+1} \oplus V_{n} \oplus V_{n-1}$ (12) implies that 


$$
X_{j}\left|\bar{n}>=P_{n+1} X_{j}\right| \bar{n}>+P_{n} X_{j}\left|\bar{n}>+P_{n-1} X_{j}\right| \bar{n}>;
$$

and, since $\mid \bar{n}>\in V_{n}$ is arbitrary, this is equivalent to (11).

Now define the following operators:

$$
\begin{gathered}
D_{n}^{+}(j):=\left.P_{n+1} X_{j} P_{n}\right|_{V_{n}} \in \mathcal{B}\left(V_{n}, V_{n+1}\right) \\
D_{n}^{0}(j):=\left.P_{n} X_{j} P_{n}\right|_{V_{n}} \in \mathcal{B}\left(V_{n}, V_{n}\right)=\mathcal{B}\left(V_{n}\right) \\
D_{n}^{-}(j):=\left.P_{n-1} X_{j} P_{n}\right|_{V_{n}} \in \mathcal{B}\left(V_{n}, V_{n-1}\right)
\end{gathered}
$$

Given any orthonormal basis $(\mid \bar{n}>)$ of $V_{n}$, we can write the finite dimensional operators (13), (14), (15) as matrices:

$$
\begin{aligned}
& D_{n}^{0}(j)=\sum_{\bar{m}, \bar{n}}|\bar{m}><\bar{m}| D_{n}^{0}(j) \bar{n}><\bar{n}\left|=: \sum_{|\bar{m}|=|\bar{n}|=n} D_{\bar{m}, \bar{n}}^{0}(j)\right| \bar{m}><\bar{n} \mid \\
& D_{n}^{+}(j)=\sum_{\bar{n}, \bar{m}}|\bar{m}><\bar{m}| D_{n}^{+}(j) \bar{n}><\bar{n} \mid=: \\
& D_{n}^{-}(j)=\sum_{|\bar{n}|=n,|\bar{m}|=n+1} D_{\bar{n}, \bar{m}}^{+}(j)|\bar{m}><\bar{n}|
\end{aligned}
$$

where, if $\bar{n}=\left(n_{1}, \ldots, n_{d}\right)$, we use the notation $|\bar{n}|=n_{1}+\ldots+n_{d}$.

We note that

$$
\begin{gathered}
D_{n}^{+}(j) \text { is represented by an } r_{n}^{d} \times r_{n+1}^{d} \text { matrix } \\
D_{n}^{0}(j) \text { is represented by an } r_{n}^{d} \times r_{n}^{d} \text { matrix } \\
D_{n}^{-}(j) \text { is represented by an } r_{n}^{d} \times r_{n-1}^{d} \text { matrix }
\end{gathered}
$$


Lemma 3 . The operators (13), (14), (15) satisfy the following relations:

$$
\begin{gathered}
D_{n+1}^{+}(i) D_{n}^{+}(j)=D_{n}^{+}(j) D_{n+1}^{+}(i) \\
D_{n+1}^{0}(i) D_{n}^{+}(j)+D_{n}^{+}(i) D_{n}^{0}(j)=D_{n+1}^{0}(j) D_{n}^{+}(i)+D_{n}^{+}(j) D_{n}^{0}(i) \\
D_{n-1}^{+}(i) D_{n}^{-}(j)+D_{n}^{0}(i) D_{n}^{0}(j)+D_{n+1}^{-}(i) D_{n}^{+}(j)= \\
D_{n-1}^{+}(j) D_{n}^{-}(i)+D_{n}^{0}(i) D_{n}^{0}(j)+D_{n+1}^{-}(j) D_{n}^{+}(i)
\end{gathered}
$$

for $i \neq j, 1 \leq i, j \leq d$ and $n \geq 0$, where $D_{-1}^{+}(i)=0$.

Proof. We have

$$
D_{n+1}^{+}(j) D_{n}^{+}(i)=\left.\left.P_{n+2} X_{j} P_{n+1}\right|_{V_{n+1}} P_{n+1} X_{i} P_{n}\right|_{V_{n}}=\left.P_{n+2} X_{j} P_{n+1} X_{i} P_{n}\right|_{V_{n}}
$$

Thus, exchanging $i$ and $j$ :

$$
D_{n+1}^{+}(i) D_{n}^{+}(j)=\left.P_{n+2} X_{i} P_{n+1} X_{j} P_{n}\right|_{V_{n}}
$$

On the other hand, since $X_{i} X_{j}=X_{j} X_{i}$, it follows that

$$
P_{n+2} X_{i} X_{j} P_{n}=P_{n+2} X_{j} X_{i} P_{n}
$$

Using (11) we see that the left hand side of (24) is equal to

$$
P_{n+2} X_{i} P_{n+1} X_{j} P_{n}+P_{n+2} X_{i} P_{n} X_{j} P_{n}+P_{n+2} X_{i} P_{n-1} X_{j} P_{n}
$$

and the right hand side to

$$
P_{n+2} X_{j} P_{n+1} X_{i} P_{n}+P_{n+2} X_{j} P_{n} X_{i} P_{n}+P_{n+2} X_{j} P_{n-1} X_{i} P_{n}
$$

Since

$P_{n+2} X_{i} P_{n} X_{j} P_{n}=P_{n+2} X_{j} P_{n-1} X_{i} P_{n}=P_{n+2} X_{j} P_{n} X_{i} P_{n}=P_{n+2} X_{i} P_{n-1} X_{j} P_{n}=0$ 
this is equivalent to

$$
P_{n+2} X_{i} P_{n+1} X_{j} P_{n}=P_{n+2} X_{j} P_{n} X_{i} P_{n}
$$

Therefore (22) and (23) are equal, which proves (19).

In a similar way we observe that

$$
P_{n+1} X_{i} X_{j} P_{n}=P_{n+1} X_{j} X_{i} P_{n}
$$

is equivalent to

$$
\begin{aligned}
& P_{n+1} X_{i} P_{n+1} X_{j} P_{n}+P_{n+1} X_{i} P_{n} X_{j} P_{n}+P_{n+1} X_{i} P_{n-1} X_{j} P_{n}= \\
& P_{n+1} X_{j} P_{n+1} X_{i} P_{n}+P_{n+1} X_{j} P_{n} X_{i} P_{n}+P_{n+1} X_{j} P_{n-1} X_{i} P_{n}
\end{aligned}
$$

Since

$$
P_{n+1} X_{i} P_{n-1} X_{j} P_{n}=P_{n+1} X_{j} P_{n-1} X_{i} P_{n}=0
$$

the relation (25) implies

$$
D_{n+1}^{0}(i) D_{n}^{+}(j)+D_{n}^{+}(i) D_{n}^{0}(j)=D_{n+1}^{0}(j) D_{n}^{+}(i)+D_{n}^{+}(j) D_{n}^{0}(i)
$$

which proves $(20)$.

To prove (21) we apply the recurrence relation to the two sides of the equality

$$
P_{n} X_{i} X_{j} P_{n}=P_{n} X_{j} X_{i} P_{n}
$$

and argue as above.

Lemma 4 . The operators $D_{n}^{+}(j), D_{n}^{-}(j), D_{n}^{0}(j)$ are such that

$$
\left(D_{n}^{+}(j)\right)^{*}=D_{n+1}^{-} \quad ; \quad\left(D_{n}^{0}(j)\right)^{*}=D_{n}^{0}(j)
$$

Proof.

$$
\begin{gathered}
\left(D_{n}^{0}(j)\right)^{*}=\left(P_{n} X_{j} P_{n}\right)^{*}=P_{n} X_{j} P_{n}=D_{n}^{0}(j) \\
\left(D_{n}^{+}(j)\right)^{*}=\left(P_{n+1} X_{j} P_{n}\right)^{*}=P_{n} X_{j} P_{n+1}=D_{n+1}^{-}
\end{gathered}
$$


Lemma 5 . If $\mu$ is generic, then $D_{n}^{+}(j)$ is injective for any $n \in \mathbf{N}$ and $j=1, \ldots, d$.

Proof. If there exists $\bar{n} \in V_{n}$ such that

$$
P_{n+1} X_{j} \bar{n}=0
$$

then

$$
X_{j} \bar{n}=P_{n} X_{j} \bar{n}+P_{n-1} X_{j} \bar{n}
$$

But the left hand side of this equality is a polynomial of degree $n+1$ and the right hand side is a polynomial of degree $n$. Because of the genericity of $\mu$ they can be equal if and only if they are both zero. Also because of genericity $X_{j}$ is invertible in $\mathcal{H}_{\mu}$, hence

$$
X_{j} \bar{n}=0 \Longrightarrow \bar{n}=0
$$

It follows that $P_{n+1} X_{j} P_{n}=D_{n}^{+}(j)$ is injective.

Lemma 6 . For each $n \in \mathbf{N}$, the family $D_{n}^{+}(1), \ldots, D_{n}^{+}(d): V_{n} \longrightarrow V_{n+1}$ is surjective, in the sense that the linear span of the ranges of $D_{n}^{+}(j)$ is the whole of $V_{n+1}$.

Proof. Let $\xi \in V_{n+1}$ be an element orthogonal to the range of all $D_{n}^{+}(j)$ 's $(j=1, \ldots, d)$. Then for any $\bar{n} \in V_{n}$ and for any $j=1, \ldots, d$

$$
0=<\xi, D_{n}^{+}(j) \bar{n}>=<\xi, P_{n+1} X_{j} \bar{n}>=<\xi, X_{j} \bar{n}>
$$

But, by definition of $V_{n}$, it also follows that $\xi \perp X_{j} V_{k}$ for any $k \leq n-1$. By taking linear combinations we see that $\xi$ is orthogonal to all polynomials of the form $X_{j} q_{n}$ where $q_{n} \in P_{n}$. But, taking linear combinations of these, we obtain all possible polynomials of degree $n+1$ without constant term. This contradicts the fact that $\xi \in V_{n+1}$.

\section{$2 \quad$ Interacting Fock spaces}

Let us recall the definition of interacting Fock space. We use here the definition introduced by [AcSk99] which, although equivalent to the original one of [AcLu92], [AcLuVo97], is more suitable for our purposes. 
Definition 4 Let $\mathcal{H}_{1}^{0}$ be a pre-Hilbert space. An interacting Fock space based on $\mathcal{H}_{1}^{0}$ is an Hilbert space $\mathcal{H}$, with a gradation indexed by $\mathbf{N}$

$$
\mathcal{H}=\bigoplus_{n \geq 0} \mathcal{H}_{n}=C \cdot \Phi \oplus \bigoplus_{n \geq 1} \mathcal{H}_{n}
$$

and with the following property.

$\forall v \in \mathcal{H}_{1}^{0}$, there exists a densely defined linear operator $a^{+}(v)$ on $\mathcal{H}$ such that:

(i) the map $\quad v \in \mathcal{H}_{1}^{0} \mapsto a^{+}(v)$ is complex linear.

(ii) $\forall n \in \mathbf{N}$ the set

$$
\left\{a^{+}\left(v_{n}\right) \ldots a^{+}\left(v_{1}\right) \Phi: v_{1}, \ldots, v_{n} \in \mathcal{H}_{1}^{0}\right\}
$$

is contained in the domain $D\left(a^{+}(v)\right)$ of $a^{+}(v)$. For fixed $n \in \mathbf{N}$, we will denote $\mathcal{N}_{n}$ the linear subspace (algebraically) generated by the vectors (28).

(iii) The union of all the $\mathcal{N}_{n}(n \in \mathbf{N})$ is a dense subspace of $\mathcal{H}$.

(iv) $a^{+}(v)$ maps $\mathcal{N}_{n}$ onto $\mathcal{N}_{n+1}$.

$(v)$ Each $a^{+}(v)$ has an adjoint $a(v)$ defined on $\mathcal{N}:=\bigcup_{n} \mathcal{N}_{n}$.

Remark. The direct sum in (27) is in the Hilbert space sense. We will denote

$$
\mathcal{H}^{0}=\bigoplus_{n \geq 0}^{\text {algebraic }} \mathcal{H}_{n} \quad ; \quad \mathcal{H}_{0}=C \cdot \Phi
$$

the algebraic direct sum, i.e. the subspace of $\mathcal{H}$ of the vectors which have a non zero component only on a finite number of spaces $\mathcal{H}_{n}$.

Now let us fix the following choices:

$$
\mathcal{H}=\mathcal{H}_{\mu} \quad ; \quad \mathcal{H}_{\mu}^{0}=\bigoplus_{n}^{\text {algebraic }} \mathcal{H}_{\mu} \quad ; \quad \mathcal{H}_{1}^{0}=C^{d} \quad ; \quad \mathcal{H}_{n}=V_{n}^{d}
$$

where the operators $a_{j}^{+}$are defined by the following: 
Proposition 1 In the notation (10) let, for $n \in \mathbf{N},|\bar{n}>=| n_{1}, \ldots, n_{d}>$ denote an arbitrary orthonormal basis of $V_{n}$ and let $D_{\bar{m}, n}^{\epsilon}(j)$ denote the matrices in this basis, of the operators $D_{n}^{\epsilon}(j)(\epsilon=+,-, 0, n \in \mathbf{N})$ as defined by (16) , (17), (18). Then:

(i) For each $j=1, \ldots, d$ the operator $a_{j}^{+}$, defined on the vectors $\mid n_{1}, \ldots, n_{d}>$ by

$$
a_{j}^{+}\left|n_{1}, \ldots, n_{d}>=D_{n}^{+}(j)\right| \bar{n}>=\sum_{\left|\bar{m}_{+}\right|=n+1} D_{\bar{n}, m_{+}}^{+}(j) \mid \bar{m}_{+}>
$$

has an adjoint, also defined on the vectors $\mid n_{1}, \ldots, n_{d}>$ by

$$
\begin{gathered}
a_{j}^{-} \mid 0, \ldots, 0>=0 \\
a_{j}^{-}\left|\bar{n}>=D_{n}^{-}(j) \bar{n}=\sum_{\left|\bar{m}_{-}\right|=n-1} D_{\bar{n}, \bar{m}_{-}}^{-}(j)\right| \bar{m}_{-}>
\end{gathered}
$$

(ii) The operators $a_{j}^{0}(j=1, \ldots, d)$ defined by

$$
a_{j}^{0}\left|\bar{n}>=\sum_{|\bar{m}|=n} D_{\bar{n}, \bar{m}}^{0}(j)\right| \bar{m}>
$$

is symmetric on $H_{\mu}^{0}$.

(iii) The following identity holds on $H_{\mu}^{0}$ :

$$
X_{j}:=a_{j}^{+}+a_{j}^{0}+a_{j}^{-}, j=1, \ldots, d
$$

Remark. Notice that that definition of the operators $a_{j}^{+}$depends on the choice of the orthonormal basis $\left|n_{1}, \ldots, n_{d}\right\rangle$.

An intrinsic definition is possible, but makes the intuitive connection with ordinary multiplication operators more obscure.

Proof. Since, for each $\bar{n} \neq \overline{0}, a_{j}^{+} \mid \bar{n}>$ is always in some $V_{k}$ with $k \geq 1$, one has

$$
<\overline{0}, a_{j}^{+} \bar{n}>=0
$$

Moreover since, by definition,

$$
\left.a^{+}(j)\right|_{V_{n}}=D_{n}^{+}(j)=P_{n+1} X_{j} P_{n}
$$

and

$$
\left.a^{-}(j)\right|_{V_{n+1}}=D_{n+1}^{-}(j)
$$


it follows that

$$
\left(D_{n}^{+}(j)\right)^{*}=P_{n} X_{j} P_{n+1}=a_{n+1}^{-}(j),
$$

which means that $a^{-}(j)$ is the adjoint of $a^{+}(j)$ on $H_{\mu}^{0}$. Similarly

$$
\left.a^{0}(j)\right|_{V_{n}}=D_{n}^{0}(j)=P_{n} X_{j} P_{n}=\left(P_{n} X_{j} P_{n}\right)^{*}=\left(D_{n}^{0}(j)\right)^{*}
$$

Therefore the operator $a_{j}^{0}$ is symmetric on $H_{\mu}^{0}$. For each $n \in \mathbf{N}$ the identity

$$
X_{j} P_{n}=\left(a_{j}^{+}+a_{j}^{0}+a_{j}^{-}\right) P_{n}
$$

follows from the definition of the $D_{n}^{\epsilon}(j), \epsilon=+,-, 0$ and this implies (34).

Conversely:

Theorem 2 Let be given a Hilbert space $\mathcal{H}$ with a $\mathbf{N}$-gradation

$$
\mathcal{H}=\bigoplus_{n \in \mathbf{N}} V_{n} \quad ; \quad V_{0}=C \cdot \Phi
$$

such that

$$
V_{n} \equiv C^{r_{n}^{d}} \quad \forall n \in N
$$

and let be given:

(i) d sequences of (finite dimensional) operators

$$
\begin{gathered}
D_{n}^{+}(j) \in \mathcal{B}\left(V_{n}, V_{n+1}\right) \\
D_{n}^{0}(j) \in \mathcal{B}\left(V_{n}, V_{n}\right) \\
D_{n}^{-}(j) \in \mathcal{B}\left(V_{n}, V_{n-1}\right)
\end{gathered}
$$

satisfying the conditions (19), (20), (21).

(ii) For each $n \in \mathbf{N}$ an orthonormal basis of $V_{n}$, denoted

$$
|\bar{n}>=| n_{1}, \ldots n_{d}>\quad ; \quad|\bar{n}|:=\sum_{j=1}^{d} n_{j}=n
$$

Then, on $\mathcal{H}$, there exists a structure of interacting Fock space over $C^{d}$, with creation and annihilation operators given by (30), (31), (33) respectively. 
If, in addition the commutativity relations (19), (20), (21) are satisfied, then the operators

$$
X_{j}:=a_{j}^{+}+a_{j}^{0}+a_{j}^{-} \quad ; \quad j=1, \ldots, d
$$

are a commuting family of symmetric operators satisfying the relations (11), $P_{n}$ being the orthonogonal projection onto $V_{n}$. Moreover $\Phi$ is a cyclic vector for the polynomial algebra generated by the $X_{j}$ and the identity.

Proof. Let us fix arbitrarily, for each $n \in \mathbf{N}$, an orthonormal basis $(\mid \bar{n}>)$ of $V_{n}$. Define the operators $a_{j}^{+}, a_{j}^{-}, a_{j}^{0}$ by $(30),(32),(33),(j=1, \ldots, d)$ respectively.

The symmetry of the $a_{j}^{0}$ comes from the fact that the $D_{n}^{0}(j)$ are symmetric and the fact that $a_{j}^{-}$is the adjoint of $a_{j}^{+}$comes from the relation $D_{n}^{-}(j)=$ $\left(D_{n}^{+}(j)\right)^{*}$ as in the proof of Proposition (1). This implies that the operators $X_{j}$, defined by (37) are symmetric on $H_{\mu}^{0}$ and the relation (11) holds.

The commutativity of the family $\left(X_{1}, \ldots, X_{d}\right)$ comes from the relations (19), (20), (21) as follows.

For each $i, j=1, \ldots, d$ and $n \in \mathbf{N}$ we have

$$
\begin{gathered}
X_{i} X_{j} \mid \bar{n}>= \\
\left(a^{+}(i)+a^{0}(i)+a^{-}(i)\right)\left(a^{+}(j)+a^{0}(j)+a^{-}(j)\right) \mid \bar{n}>= \\
\left(a^{+}(i) a^{+}(i)+a^{+}(i) a^{+}(0)+a^{+}(i) a^{+}(-)\right) \mid \bar{n}>+ \\
\left(a^{0}(i) a^{+}(i)+a^{0}(i) a^{+}(0)+a^{0}(i) a^{+}(-)\right) \mid \bar{n}>+ \\
\left(a^{-}(i) a^{+}(i)+a^{-}(i) a^{+}(0)+a^{-}(i) a^{+}(-)\right) \mid \bar{n}>= \\
\left(D_{n+1}^{+}(i) D_{n}^{+}(j)+D_{n}^{+}(i) D_{n}^{0}(j)+\left(D_{n-1}^{+}(i) D_{n}^{-}(j)\right) \mid \bar{n}>+\right. \\
\left(D_{n+1}^{0}(i) D_{n}^{+}(j)+D_{n}^{0}(i) D_{n}^{0}(j)+\left(D_{n-1}^{0}(i) D_{n}^{-}(j)\right) \mid \bar{n}>+\right. \\
\left(D_{n+1}^{-}(i) D_{n}^{+}(j)+D_{n}^{-}(i) D_{n}^{0}(j)+\left(D_{n-1}^{-}(i) D_{n}^{-}(j)\right) \mid \bar{n}>=\right.
\end{gathered}
$$




$$
\begin{gathered}
D_{n+1}^{+}(i) D_{n}^{+}(j)\left|\bar{n}>+\left(D_{n-1}^{+}(i) D_{n}^{+}(j)+D_{n}^{0}(i) D_{n}^{0}(j)+D_{n+1}^{-}(i) D_{n}^{+}(j)\right)\right| \bar{n}>+ \\
\left(D_{n}^{-}(i) D_{n}^{0}(j)+D_{n-1}^{0}(i) D_{n}^{-}(j)+D_{n-1}^{-}(i) D_{n}^{-}(j)\right) \mid \bar{n}>+ \\
\left(D_{n+1}^{0}(i) D_{n}^{+}(j)+D_{n}^{+}(i) D_{n}^{0}(j)\right) \mid \bar{n}>
\end{gathered}
$$

The relations (19), (20), (21) imply that the above expression is equal to

$$
X_{j} X_{i} \mid \bar{n}>
$$

which proves the commutativity.

By assumption, each $D_{n}^{+}(j)$ is injective. Therefore the operators $a_{j}^{+}$are injective.

By assumption, for each $n \in \mathbf{N}$ the linear span of the ranges of the operators $D_{n}^{+}(j)(j=1, \ldots, d)$ is the whole of $V_{n}$. Therefore the vectors of the form $(28)$ are total in $\mathcal{H}$. Finally denote $\mathcal{P}_{D}$ the (polynomial) algebra generated by the $X_{j}$ and the identity. Then $\Phi \in \mathcal{P}_{D} \cdot \Phi$ and since, due to (31)

$$
\left(a_{j}^{+}+a_{j}^{0}+a_{j}^{-}\right) \Phi=a_{j}^{+} \Phi+D_{0}^{0}(j) \Phi
$$

$D_{0}^{0}(j)$ is a constant, also the vectors of the form $a_{j}^{+} \Phi$ are in $\mathcal{P}_{D} \cdot \Phi$. Now suppose, by induction, that

$$
\bigoplus_{k=0}^{n} V_{k} \subseteq \mathcal{P}_{D} \cdot \Phi
$$

Then, because of the relation (11), for any $\mid \bar{n}>\in V_{n}$, one has

$$
X_{j}\left|\bar{n}>=P_{n+1} X_{j}\right| \bar{n}>+P_{n} X_{j}\left|\bar{n}>+P_{n-1} X_{j}\right| \bar{n}>
$$

and, by the induction assumption

$$
P_{n} X_{j}\left|\bar{n}>+P_{n-1} X_{j}\right| \bar{n}>\in \bigoplus_{k=0}^{n} V_{k} \subseteq \mathcal{P}_{D} \cdot \Phi
$$

It follows that also 


$$
X_{j} \mid \bar{n}>-\left(P_{n} X_{j}\left|\bar{n}>+P_{n-1} X_{j}\right| \bar{n}>=a_{j}^{+}\left|\bar{n}>=D_{n}^{+}(j)\right| \bar{n}>\in \mathcal{P}_{D} \cdot \Phi\right.
$$

The surjectivity condition then implies that $V_{n+1} \subseteq \mathcal{P}_{D} \cdot \Phi$ and therefore the thesis follows by induction. 
The interacting Fock space defined by the theorem (??) will be denoted

$$
\mathcal{H}_{D}
$$

Theorem 3 Suppose all the conditions of Theorem 2 are fullfilled. Then there exists a state $\mu$ on the ${ }^{*}$-algebra $\mathcal{A}$ of the polynomials in $d$ variables such that, denoting $L^{2}\left(\boldsymbol{R}^{d}, \mu\right)$ the GNS space of the pair $\{\mathcal{A}, \mu\}$, there exists an unitary isomorphism

$$
U: L^{2}\left(\boldsymbol{R}^{d}, \mu\right) \longrightarrow \mathcal{H}_{D}
$$

such that for any $j=1, \ldots, d$

$$
\begin{gathered}
U X_{j} U^{*}=a_{j}^{+}+a_{j}^{0}+a_{j}^{-} \\
U 1=\Phi
\end{gathered}
$$

Proof: We define

$$
\mu(P)=<0, P\left(X_{1}, \ldots, X_{d}\right) \mid 0>, \quad \text { for each polynomial } \quad P \in \mathcal{A}
$$

It is clear that $\mu$ is a state on $\mathcal{A}$. Define

$$
U: L^{2}\left(\mathbf{R}^{d}, \mu\right) \longrightarrow \mathcal{H}_{D}
$$

by

$$
U(P)=P\left(X_{1}, \ldots, X_{d}\right) \overline{0}, \quad \text { for each polynomial } \quad P \in \mathcal{A}
$$

It is easy to verify that $U$ is an unitary isomorphism wich satisfies (39).

\section{Bibliography}

[AcBo98] Luigi Accardi, Marek Bożejko: Interacting Fock spaces and Gaussianization of probability measures Infinite dimensional analysis, quantum probability and related topics, 2, N. 4 (1998) Volterra Preprint (1997) N. 321

[AcSk98] L. Accardi and M. Skeide: Interacting Fock space versus full Fock module, Volterra Preprint N. 328 (1998) 
[AcLuVo97b] Accardi L., Lu Y.G., I. Volovich The QED Hilbert module and Interacting Fock spaces. Publications of IIAS (Kyoto) (1997)

[AcLu92b] Accardi L., Lu Y.G.: The Wigner Semi-circle Law in Quantum Electro Dynamics, Commun. Math. Phys. 180 (1996) 605-632 Volterra preprint N.126 (1992)

[Asai01] Nobuhiro ASAI: Analytic Characterization of One-mode Interacting Fock Space, IDA-QP 4 (2001) 409-415

[Das01a] Das P.K.: Coherent states and squeezed states in interacting Fock space Preprint ((2001)

[Das01b] Das P.K.: A phase distribution in interacting Fock space Preprint $((2001)$

[DuXu01] C.F. Dunkl, Y. Xu: Orthogonal polynomials of several variables, Cambridge University Press (2001)

[Hash97] Hashimoto Y.: Deformations of the semi-circle law derived from random walks on free groups, Preprint (1997)

[HaHoOb01] Hashimoto Y., Hora A., Obata N.: Central limit theorems for large graphs: a method of quantum decomposition, Preprint (2001)

[HaObTa01] Obata N., Hashimoto Y., Tabei N.: A quantum aspect of asymptotic spectral analysis of large Hamming graphs, Preprint (2001)

[KoSw94] R. Koekoek and R.F. Swarttouw: The Askey-scheme of hypergeometric orthogonal polynomials and its $q$-analogue, Technical Report 94-05 Technical University of Delft (1994) Preprint math.CA/9602214 also available from twi.tudelft.nl in directory/pub/publications/tech-reports/(1994)

[Kowa82a] M.A. Kowalski, The recursion formulas for polynomials in $\mathrm{n}$ variables, SIAM J. Math. Anal. 13 (1982) 309-315

[Kowa82b] M.A. Kowalski: Orthogonality and recursion formulas for polynomials in n variables, SIAM J. Math. Anal. 13 (1982) 316-323

[KrShe67] H.L. Krall, I.M. Sheffer, Orthogonal polynomials in two variables, Ann. Mat. Pura Appl. 76, no. 4 (1967) 325-376

[Koor90] Koornwinder, T.H.: Orthogonal polynomials in connection with quantum groups, Nevai, P. (ed.) Orthogonal Polynomials, Kluwer Acad. Publ. (1990) 257-292

[Sak71] S. Sakai. $C^{*}$-Algebras and $W^{*}$-algebras. Springer (1971)

[Szeg] H. Szego: Orthogonal polynomials, 4th ed. Amer. Math. Soc. Colloq. Publ. vol.23, Providence, R.I.

[Xu97a] Y. Xu: On orthogonal polynomials in several variables, Fields Institute Communications, Vol. 14 (1997) 247-270 
[Xu93] Y. Xu: Unbounded commuting operators and multivariable orthogonal polynomials, Proc. Amer. Math. Soc. 119 (1993) 1223-12231 